\title{
RUPTURA DEL SENO MARGINAL DE LA PLACENTA Y OTRAS HEMORRAGIAS PLACENTARIAS.
}

\section{Estudio Comparativo de 118 Casos.}

Dr. Bernardo Handszer G.

Dr. Luis A. Alba Palacios

Dra. Elsa Valderrama $M$.

El propósito de este estudio es presentar las similitudes, diferencias y relaciones que existen entre las entidades denominadas como Abruptio Placentae, Placenta Previa y Ruptura del Seno marginal de la placenta, con la intención de hacer más fácil el diagnóstico, manejo y diferenciación de éstas.

\section{Material y Métodos}

Se utilizaron para este estudio 118 casos de hemorragia del último trimestre del embarazo, en forma prospectiva, tomadas de las pacientes que ingresaron al Instituto Materno Infantil "Concepción Villaveces de Acosta", de Bogotá en el lapso comprendido entre el $1^{\circ}$ de Mayo y el 31 de Diciembre de 1965.

Se analizaron sus condiciones clínicas, signos y síntomas presentes al ingreso y durante su estadía hasta ocurrido el parto.

Los datos analizados al ingreso de las pacientes, fueron los siguientes:

a) Iniciación de la hemorragia, (anteparto, intraparto, en el período de dilatación o en el período expulsivo). b) Cantidad de la hemorragia: abundante (500 cc. o más); moderada (200 a 400 cc.); escasa (200 cc. o menos).

c) Alteraciones de la dinámica uterina (hipertonía, polisistolia).

d) Sintomatología dolorosa.

e) Signología de toxemia.

f) Estado fetal al ingreso (bueno, regular con signos de sufrimiento, muerto).

g) Diagnóstico de ingreso.

Hemos incluído todos los casos en los cuales hubo tal hemorragia que la paciente tuvo que ser clasificada, colocada en condiciones especiales de expectativa armada y practicado un examen genital cuidadoso.

En lo que se refiere al abruptio y ruptura del Seno marginal, se utilizaron criterios diagnósticos ceñidos al aspecto anatómico de las placentas, y el clínico para el diagnóstico de placenta previa.

Se descartó el diagnóstico de Placenta Previa al no encontrar la placenta en el examen cérvico-vaginal; el 
Abruptio también se descartó como entidad cuando el aspecto anatómico de la placenta ni el cuadro clínico pudieron ser considerados diagnósticos.

Este criterio inflexible sirve para prevenir el hecho de que el diagnóstico de ruptura de Seno marginal, sea un diagnóstico de eliminación $\mathrm{o}$ de exclusión. Una aceptación de este criterio podría ser un mal comienzo para una entidad cuya existencia se ha pretendido demostrar.

El criterio de base para el diagnóstico de Abruptio se fundamentó en el hallazgo de uno o más de los siguientes signos:

a) Un coágulo retroplacentario mensurable.

b) Una depresión en la superficie materna de la placenta causada por ese coágulo.

c) Cambio de color y textura inequívocos de la superficie materna de la placenta.

d) Hallazgo de un infarto agudo o subagudo en la placenta cerca del sitio de la hemorragia.

El Abruptio al tomar únicamente un área localizada del margen de la placenta, puede producir hemorragia que simula la producida por la ruptura del Seno marginal o viceversa.

Es frecuente observar manifestaciones de toxemia asociadas con esta entidad.

Los casos de ruptura antenatal del seno marginal, el estudio de la placenta muestra coágulos recientes 0 antiguos, adherentes a una porción del margen de la placenta encima de un desgarro del seno marginal, ocasionalmente extendiéndose sobre las membranas adyacentes o cubriendo una pequeña porción de la superficie materna sobre los cotiledones margi- nales. La identificación de una ruptura en la pared del seno no es patognomónica de hemorragia si no hay un trombo sobre el sitio de ésta, en continuidad con un coágulo en el interior del mismo.

Si al examen se observa que el coágulo hubiese podido estar entre la superficie materna y el útero, el diagnóstico tomado es el de Abruptio.

El diagnóstico de placenta previa se comprobó siempre por el examen vaginal.

Anatómicamente, una ruptura central de las membranas presumiblemente elimina la placenta previa. Cuando el sitio de ruptura es marginal o lateral, la determinación de la implantación baja depende más del examen vaginal que de otros hallazgos, lógicamente estando la ruptura de las membranas cerca de la ruptura del seno marginal.

Las placentas fueron recogidas cuidadosamente y observadas en su totalidad en fresco, inmediatamente después del parto, colocándolas sobre un papel extendido. Después de medir la distancia del sitio de ruptura de las membranas al borde de la placenta se amplió la brecha de éstas, para estudiar la cara materna y sus modificaciones.

\section{Resultados}

CUADRO N: 1

INICIACION DE LA HEMORRAGIA

\begin{tabular}{lrc}
\hline & Casos & Porcentaje \\
\hline Anteparto & 66 & 56,0 \\
Intraparto & 44 & 37,2 \\
Expulsivo & 8 & 6,8 \\
TOTAL & 118 & 100,0 \\
\hline
\end{tabular}

De los 118 casos que fueron tomados para estudio, en el $56 \%$ se presentó la hemorragia en el período an- 
teparto, $37,2 \%$ intraparto y el $6,8 \%$ durante la fase expulsiva.

CUADRO № 2

\section{SIGNOS Y SINTOMAS ASOCIADOS}

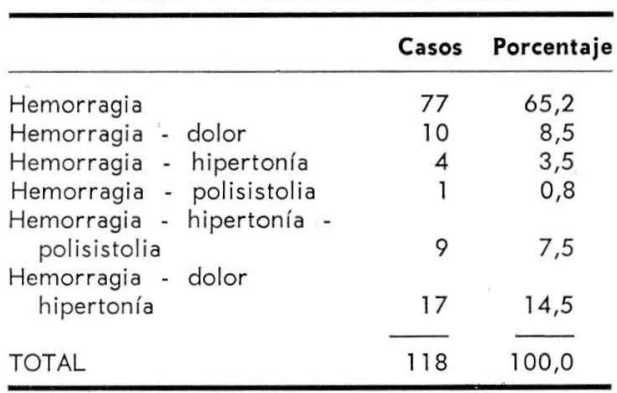

La hemorragia fue signo predominante presentándose en 77 casos $(65,2 \%)$ mientras que se asoció con dolor e hipertonía en 17 casos y solamente con dolor en 10 casos. La asociación con hipertonía y polisistolia se observó en 9 casos.
CUADRO NN 3

\section{CANTIDAD DE LA HEMORRAGIA}

\begin{tabular}{|c|c|c|}
\hline & Casos & Porcentaje \\
\hline Abundante (500 cc. o más) & 29 & 24,5 \\
\hline Moderada (200 a 400 cc.) & 44 & 37,2 \\
\hline Escasa (200 cc. o menos) & 45 & 38,3 \\
\hline TOTAL & 118 & 100,0 \\
\hline
\end{tabular}

La cantidad de hemorragia fue escasa en 45 casos, moderada en 44 y abundante en 29.

\section{CUADRO № 4} ESTADO FETAL AL INGRESO

\begin{tabular}{|c|c|c|}
\hline & Casos & Porcentaje \\
\hline $\begin{array}{l}\text { Bueno } \\
\text { Regular (Signos de }\end{array}$ & 79 & 67,0 \\
\hline sufrimiento) & 18 & 15,2 \\
\hline Muertos & 21 & 17,8 \\
\hline TOTAL & 118 & 100,0 \\
\hline
\end{tabular}

\section{CUADRO N: 5}

\section{DIAGNOSTICOS DE INGRESO $Y$ DEFINITIVOS}

\begin{tabular}{|c|c|c|c|c|}
\hline \multirow[b]{2}{*}{ Diagnóstico } & \multicolumn{2}{|c|}{ Ingreso } & \multicolumn{2}{|c|}{ Definitivo } \\
\hline & Casos & $\%$ & Casos & $\%$ \\
\hline Placenta previa central total & 12 & 10,2 & 12 & 10,2 \\
\hline Placenta previa central parcial & 8 & 6,7 & 8 & 6,7 \\
\hline Placenta previa marginal & 10 & 8,5 & 10 & 8,5 \\
\hline Placenta baja & 14 & 11,9 & - & - \\
\hline Ruptura de Seno marginal & 27 & 23,0 & 41 & 34,8 \\
\hline Abruptio placentae & 35 & 29,6 & 33 & 28,9 \\
\hline Poliposis cervical & 2 & 1,6 & 2 & 1,6 \\
\hline Sin diagnóstico & 10 & 8,5 & - & - \\
\hline Hemorragia de causa indeterminada & - & - & 12 & 10,2 \\
\hline
\end{tabular}

El estado fetal al ingreso fue considerado bueno en 79 casos, regular o co nsignos de sufrimiento en $18 \mathrm{ca}$ sos, y 21 fueron encontrados muertos.

Los diagnósticos de ingreso fueron: placenta previa central total $12 \mathrm{ca}$ sos, placenta previa central parcial 8 casos, placenta previa marginal 10 casos. Se encontraron 35 casos con sintomatología de abruptio placentae y 27 fueron interpretados como rupturas del seno marginal.

Los diagnósticos de placenta baja hechos en 14 casos no son considerados para el diagnóstico definitivo. No se pudo hacer diagnóstico de ingreso en 10 casos; en dos (2) se encontraron poliposis cervicales severas. 
Los diagnósticos definitivos fueron: 30 casos de placenta previa, 33 de abruptio placentae y 41 de ruptura del seno marginal. 12 casos fueron diagnosticados como hemorragia de causa indeterminada.

\section{CUADRO N: 6} EDAD DE LAS PACIENTES

\begin{tabular}{lrr}
\hline & Casos & Porcentaje \\
\hline 19 años o menos & 9 & 7,6 \\
20 a 30 años & 57 & 48,5 \\
31 a 40 años & 66 & 38,9 \\
41 y más & 6 & 5,0 \\
\cline { 2 - 3 } TOTAL & 118 & 100,0 \\
\hline
\end{tabular}

Estos accidentes fueron encontrados según la edad de las pacientes, en el grupo de 20 a 30 años en 57 casos, en el de 31 a 40 en 46 casos, mientras que en los de 19 o menos y 41 o más solo tuvieron 9 y 6 casos respectivamente.

CUADRO № 7

EDAD DEL EMBARAZO

\begin{tabular}{lrrr}
\hline & Casos & Porcentaje \\
\hline 20 a 27 semanas & 6 & 5,0 \\
28 a 36 semanas & 45 & 38,2 \\
37 a 40 semanas & 66 & 56,0 \\
41 semanas 0 más & 1 & 0,8 \\
\cline { 2 - 3 } TOTAL & 118 & 100,0 \\
\hline
\end{tabular}

Por edad de embarazo, fueron más frecuentes en el grupo de 37 a 40 semanas, 66 casos, siguiéndole el de 28 a 36 semanas con 45 casos.

\section{CUADRO N: 8} PARIDAD

\begin{tabular}{lcc}
\hline & Casos & Porcentaje \\
\hline Uno & 18 & 15,3 \\
Dos & 17 & 14,4 \\
Tres & 15 & 12,7 \\
Cuatro & 9 & 7,6 \\
Cinco o más & 59 & 50,0 \\
\cline { 2 - 3 } TOTAL & 118 & 100,0 \\
\hline
\end{tabular}

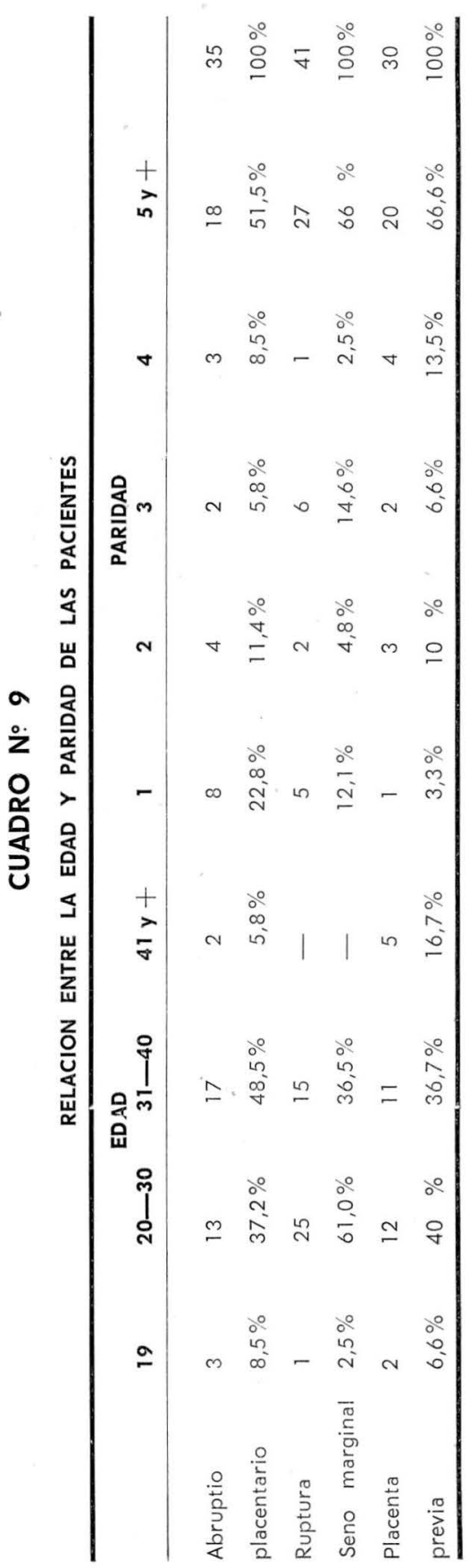


En las pacientes con 5 o más partos se obtuvieron 59 casos, mientras que en las de un parto observamos 18 casos y en las de dos partos 17 casos.

Relacionando la edad de las pacientes y la paridad encontramos en el grupo de 20 a 40 años, 30 pacientes con abruptio, 40 casos en la ruptura del seno marginal y 23 casos en la placenta previa. En cuanto a la paridad, los grupos en que se presentaron estos accidentes con mayor frecuencia fueron los de 5 o más partos, con 18 casos para el abruptio, 27 casos para la ruptura del seno marginal y 20 en la placenta previa. El total de casos observados en abruptio, ruptura del seno marginal y placenta previa fueron 35,41 y 30 respectivamente.

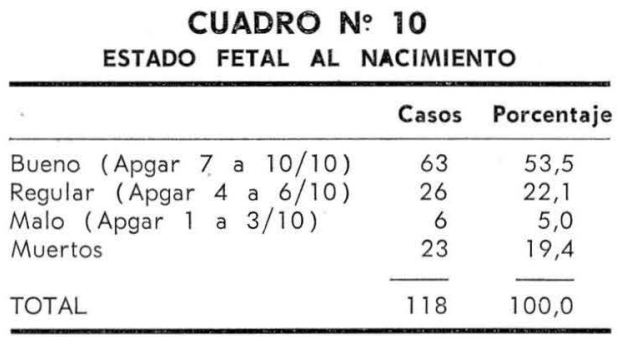

El estado fetal al nacimiento fue bueno en 63 casos calificados con Apgar 7 a 10/10; 26 nacieron en regulares condiciones, con signos de sufrimiento y Apgar 4 a 6/10. En malas condiciones, Apgar 1 a 3/10 nacieron 6. Veintitrés nacieron muertos.

\begin{tabular}{|c|c|c|}
\hline & Casos & Porcentaje \\
\hline 500 a 999 gramos & 2 & 1,6 \\
\hline 1.000 a 2.499 gramos & 47 & 39,8 \\
\hline 2.500 ○ más gramos & 65 & 55,1 \\
\hline Sin dato & 4 & 3,5 \\
\hline TOTAL & 118 & 100,0 \\
\hline
\end{tabular}

Los fetos obtenidos presentaron pesos entre 1.000 y 2.499 gramos en 47 casos, y de 2.500 o más gramos 65 casos.

CUADRO N 12

MORTALIDAD MATERNA Y FETAL

\begin{tabular}{lrc}
\hline & Casos & Porcentaje \\
\hline Mortalidad materna & 2 & 1,6 \\
Mortalidad fetal & 30 & 25,4 \\
\hline
\end{tabular}

Mortalidad materna hubo en 2 casos y fetal en 30 .

CUADRO N: 13

MODALIDAD DEL PA?TO

\begin{tabular}{lrc}
\hline & Casos & Porcentaje \\
\hline Espontáneo & 82 & 69,6 \\
Fórceps & 6 & 5,0 \\
Cesárea & 24 & 24,6 \\
Extracción podálica & 1 & 0,8 \\
TOTAL & 118 & 100,0 \\
\hline
\end{tabular}

Los partos fueron espontáneos en 82 casos, se aplicaron 6 fórceps, se practicaron 24 cesáreas y una extracción podálica.

\section{CUADRO NN 14}

DISTANCIA DE LA RUPTURA DE MEMBRANAS AL BORDE PLACENTARIO

\begin{tabular}{lrr}
\hline & Casos & Porcentaje \\
\hline De 0 a $5 \mathrm{cms}$. & 9 & 8,0 \\
De 6 a $8 \mathrm{cms}$. & 72 & 61,0 \\
De 9 a $11 \mathrm{cms}$. & 28 & 23,0 \\
De $12 \mathrm{cms} .0$ más & 9 & 8,0 \\
\cline { 2 - 2 } & 118 & 100,0 \\
\hline
\end{tabular}

El orificio producido por el desgarro de las membranas se encontró en 9 casos de 0 a $5 \mathrm{cms}$. del borde más bajo de la placenta, en 72 casos de 6 a $8 \mathrm{cms}$. en 28 casos de 9 a $11 \mathrm{cms}$. y en 9 de $12 \mathrm{cms}$. 0 más. 


\section{Comentarios:}

Es interesante observar que no hay grandes diferencias entre estas Entidades en cuanto a la signología relativa a la hemorragia pura por cuanto observamos 77 casos que corresponden al $75,2 \%$. La hemorragia más dolor e hipertonía estuvieron presentes en 17 casos $(14,7 \%)$.

Las demás asociaciones de hemorragia con dolor y polisistolia o con polisistolia sola no son de gran significancia estadística. La polisistolia sola presentó un porcentaje muy inferior a todos los demás signos observándose solamente en un caso.

Llama la atención que la hemorragia se inició en el anteparto en el $56 \%$ de los casos y un $37,2 \%$ intraparto, a diferencia de la observada en la Ruptura del Seno Marginal de la Placenta que es de $53,7 \%$ en el período intraparto.

El síntoma capital de hemorragia fuo clasificado como se explica atrás, de acuerdo con la cantidad observada al ingreso de la paciente, bien fuera en forma de sangre líquida, coágulos - la impregnación de ropas, toallas o apósitos. Fue abundante (500 cc. o más) en un 24,5\%; moderada o escasa en un grupo más o menos semejante alrededor del 38\%, $(37,2 \%$ y $38,3 \%$ respectivamente).

La cantidad promedio de hemorragia presente tuvo una incidencia de 400 cc. para los casos de Abruptio y placenta previa, mientras que para la ruptura del Seno Marginal fue de 200 cc. semejante a la encontrada por Ferguson y colaboradores.

En cuanto al estado fetal, el $67 \%$ de casos presentaron fetos en buenas condiciones al ingreso, mientras que en 21 casos, $(17,8 \%)$, se presentó muerte antenatal. Todos los casos an- teriores fueron atribuibles a la placenta previa o al abruptio. En la ruptura del Seno Marginal, la cantidad de hemorragia no tuvo incidencia aparente en la muerte fetal antenatal, al no influir sobre el estado general materno.

Tomando como base los criterios expuestos para aceptar una determinada entidad, se hizo el diagnóstico de placenta previa (Total parcial marginal) en 30 casos que corresponden al $25,4 \%$. Los diagnósticos de ingreso de placenta baja hechos en 14 casos $(11,9 \%)$ no se consideraron dentro de los diagnósticos definitivos por cuanto no hubo criterio anatómico o clínico para su aceptación, y muchas de ellas correspondieron a rupturas del seno marginal.

Algunos de estos casos no pudieron ser clasificados, $\mathrm{ni}$ al ingreso, $\mathrm{ni}$ después de atendido el parto y estudiada la placenta. Al no encontrarse alteraciones anatómicas, ni secuencias clínicas fueron clasificadas como hemorragias de origen indeterminado, que cubre el 10,2\% de los casos. La aceptación de este término, es simplemente la demostración de la imposibilidad para determinar su origen, $y$ debe tenerse en cuenta para la codificación y rotulación de los casos comprendidos en ella; no debe tomarse en ningún caso como un sentimiento de ineptitud profesional (Ferguson ).

El abruptio placentae fue diagnosticado inicialmente en el $29,6 \%$. Al revisar el material se comprobó este diagnóstico en el 28,9\%. El porcentaje restante corresponde a dos casos en los que no hubo criterio anatómico que respaldara el diagnóstico. Se hizo diagnóstico, sorprendentemente, de ruptulra del seno marginal de la placenta en 27 casos que conforman el $23 \%$ de los diagnósticos de ingre- 
so. Decimos que sorpresivo por cuanto este diagnóstico había sido hecho en el Instituto Materno Infantil, solamente en tres oportunidades en el lapso comprendido entre el 1\% de Enero de 1961 y el 31 de Abril de 1965, sin comprobación anatómica y solo basados en el cuadro clínico.

La razón fundamental de esta evolución es la motivación y el análisis más profundo de las pacientes que ingresaron con hemorragia del último trimestre del embarazo.

Llaman más aun la atención, el hecho de comprobar anatómicamente 41 casos de ruptura del seno marginal de la placenta $(34,8 \%)$, mientras que Fish y Bartholomew obtienen 50 en 147 casos de hemorragia del tercer trimestre $(33,9 \%)$. Ferguson obtuvo en tres y medio años, en el Jackson Memorial Hospital de la Universidad de Miami, 130 casos de ruptura del seno marginal, y previamente había estudiado 51 casos observados en 6 meses de 1954, en el Charity Hospital de New Orleans, (USA), entre 2.251 partos con una proporción de 1 por 68 partos. Nosotros hemos observado un caso de ruptura del seno marginal por 324 partos. Debemos tener en cuenta que su frecuencia puede ser mayor si se analiza el volumen total de pacientes ingresadas con hemorragia del último trimestre del embarazo.

Relacionando la edad de las pacientes, estas tres entidades fueron más frecuentes en el grupo de mayor actividad reproductiva, comprendido entre los 20 y los 40 años. Sin embargo hay un predominio discreto del grupo de 20 a 30 años, con un $48,5 \%$.

Comparativamente, el abruptio, la placenta previa y la ruptura del seno marginal se presentaron con mayor frecuencia entre el grupo de 37 a 40 semanas de embarazo, $56 \%$.

Estos tres desórdenes fueron igualmente frecuentes mientras fue mayor la paridad de la paciente, así observamos que en el grupo de 5 o más partos, el abruptio tomó el 51,5\%, la ruptura del seno marginal el $66 \%$ de los casos, y la placenta previa el $66,6 \%$ de los casos.

Es posible que estas Entidades deban ser consideradas como una complicación de la multiparidad.

Observamos el hallazgo de Toxemia en 26 casos de abruptio (74,2\%) y uno en la placenta previa $(3,3 \%)$. Once casos de ruptura del seno marginal presentaron signos de toxemia, $(26,8 \%)$. Este dato es muy importante por cuanto puede marcar una diferencia fundamental entre la ruptura del seno marginal y el abruptio. Esta amplia diferencia de la incidencia de toxemia entre las dos Entidades es la única disparidad reconocida que existe previamente al comienzo de los accidentes placentarios y sugiere que la toxemia puede ser un factor para determinar entre una ruptura del seno marginal y un abruptio.

Nosotros utilizamos el término Toxemia en un sentido muy amplio que incluye todas las enfermedades hipertensivas encontradas en el último trimestre del embarazo. Nelson, Brewer; Ferguson y Haton encontraron una incidencia de Toxemia de 14,4\% y $17,0 \%$ respectivamente en las pacientes con ruptura del seno marginal. El porcentaje encontrado por nosotros es bastante superior al de otros autores, pero comprensible y lógico al tener en cuenta la alta incidencia de Toxemia en nuestro medio.

Hasta el presente no se ha descrito que la ruptura del seno marginal pueda ser un riesgo o complicación 
añadido a la Toxemia, o por el contrario, que la Toxemia tenga influencia sobre la ruptura del seno marginal.

Estos desórdenes hemorrágicos revelan en conjunto un buen pronóstico fetal, habiéndose obstenido $75,6 \%$ de fetos en aceptables condiciones generales. Etn el $19,4 \%$ hubo mortalidad fo:al, de los cuales el 17,8\% murieron en el período anteparto y $1,6 \%$ intraparto. En malas condiciones, Apgar 1 a 3/10 nacieron 6 fetos $(5 \%)$. Consideramos que este buen pronóstico en cuanto a mortalidad fetal en las 3 entidades, se debe al buen diagnóstico y manejo de estos casos por parte del personal del Instituto.

Se observó una alta incidencia de prematurez, obteniéndose en el $41,4 \%$ de los casos. (Considerando los pesos de los recién nacidos).

Hubo dos casos de mortalidad materna, 1,6\%, no imputables a estas Entidades. Una de las pacientes presentó un cuadro de ruptura de seno marginal. La afección básica fue una nefrosis grave que fue la causa de la muerte. La segunda paciente presentó un abruptio placentae y murió por un accidente cerebro-vascular consecutivo a eclampsia. Ambos fetos fueron prematuros.

Igualmente, como en el punto anterior, puede recalcarse que la oportunidad de tratamiento adecuado impidió que se presentara una mortalidad materna significativa.

Se intervinieron 24 pacientes por cesárea $(24,6 \%)$, y se aplicaron fórceps en 6 casos $(5,0 \%)$. Los partos fueron espontáneos en el $69,2 \%$ de los casos y solo se hizo una extracción podálica $(0,8 \%)$.
Como complicaciones inherentes a esta entidad solamente tuvimos 4 casos de hipofibrinogenemia que cedieron con tratamiento médico y aplicación de fibrinógeno. El shock se presentó en 6 casos en los cuales la hemorragia fue muy profusa. Vale la pena anotar que en relación con la incidencia y gravedad que implica el diagnóstico del desorden hemorrágico del último trimestre del embarazo, las complicaciones fueron muy escasas. Todas las pacientes de este grupo evolucionaron en forma satisfactoria.

Se aplicaron transfusiones en 33 ca$\operatorname{sos}(27,9 \%)$.

Son comparables estos resultados con la ausencia de complicaciones observadas en la ruptura del seno marginal de la placenta.

Se requirió inducción en el 5,9\%, mientras que el $17,1 \%$ necesitaron refuerzo del trabajo con ocitócicos por trastornos en la dinámica uterina. En el $28,8 \%$ fue necesaria la sedación con meperidina, flufenazina, fenobarbital, prometazina y clorpromazina, siendo sus indicaciones básicas la Toxemia y el abruptio.

En el $69 \%$ de los casos observados se evidenció una implantación baja de la placenta al tener en cuenta la medida del sitio de ruptura de las membranas al borde más bajo de la placenta dentro del útero. Es posible que esta observación tenga una relación íntima con la presencia de estos accidentes.

\section{Hallazgos Histo-patológicos Comentarios}

En el Cuadro № 15 se analizan los diferentes cambios histopatológicos hallados en el estudio de las placentas. 
CUADRO N: 15

\section{ABRUPTIO PLACENTAE, PLACENTA PREVIA \\ $Y$ SU RELACION CON LA RUPTURA DEL SENO MARGINAL HALLAZGOS ANATOMO-PATOLOGICOS}

\begin{tabular}{lrrrrrrr}
\hline & $\begin{array}{r}\text { Coágulo } \\
\text { Marginal }\end{array}$ & $\begin{array}{c}\text { Coágulo } \\
\text { Retropl. }\end{array}$ & R. S. M. & $\begin{array}{c}\text { Infarto } \\
\text { Hemorrag. }\end{array}$ & $\begin{array}{c}\text { Desgarro } \\
\text { Tej. Plac. }\end{array}$ & $\begin{array}{c}\text { Infarto } \\
\text { Isquemic. }\end{array}$ & $\begin{array}{c}\text { Placen- } \\
\text { titis }\end{array}$ \\
\hline $\begin{array}{l}\text { Abruptio } \\
\text { placentae }\end{array}$ & $33,3 \%$ & $50,0 \%$ & $22,2 \%$ & $33,3 \%$ & $33,3 \%$ & $22,2 \%$ & $27,9 \%$ \\
$\begin{array}{l}\text { Placenta } \\
\text { previa }\end{array}$ & $50,0 \%$ & $27,3 \%$ & $22,7 \%$ & $22,7 \%$ & $27,3 \%$ & $31,8 \%$ & $27,3 \%$ \\
$\begin{array}{l}\text { Ruptura } \\
\text { seno Marg. }\end{array}$ & $100,0 \%$ & $0,0 \%$ & $100,0 \%$ & $0,0 \%$ & $0,0 \%$ & $14,5 \%$ & $44,5 \%$ \\
\hline
\end{tabular}

Los cambios más importantes que tuvimos en cuenta para clasificar los especímenes son: a) presencia de coágulo marginal o retroplacentario; b) ruptura del seno marginal; c) infartos hemorrágicos y d) desgarros del tejido placentario. Hay otros cambios que consideramos secundarios $y$ que analizaremos posteriormente.

Estos cambios esenciales los encontramos en mayor o menor grado en el abruptio placentae y en la placenta previa, en cambio en la ruptura del seno marginal lo esencial es la presencia de un coágulo sobre esta zona que puede estar en comunicación directa con coágulos presentes en la luz del seno.

Los demás cambios no se deben encontrar ya que de por sí descartaría la entidad.

\section{Abruptio placentae}

El cambio más frecuente es la presencia de un coágulo retroplacentario, $50 \%$ de los casos, ya solo o asociado a un coágulo marginal, 33,3\% de los casos, y a ruptura del seno marginal, 22,2\% de los casos. Un hallazgo frecuente en el desgarro del tejido placentario por debajo del coágulo y la presencia de infartos hemorrágicos, 33,3\% respectivamente.

\section{Placenta previa}

En la localización central total, el cambio más constante es el desgarro del tejido placentario. En las marginales es la presencia del coágulo marginal, $50 \%$ de los casos, el que puede extenderse a la superficie externa de las membranas fetales o al tejido retroplacentario, $27,3 \%$ de los casos, produciéndose adelgazamiento o destrucción de este $(27,3 \%)$, acompañándose de infarto hemorrágico, $22,3 \%$. La ruptura del seno marginal se encontró en el 22,3\%.

\section{Ruptura del seno marginal}

Los dos hallazgos únicos y constantes son, la ruptura del seno marginal y la presencia de un coágulo de mayor o menor tamaño en diferentes estados de evolución y en ocasiones continuándose con coágulos existentes dentro de la luz del seno constituyéndose este último hallazgo en signo patognomónico de la entidad. Novak describe en los casos que han evolucionado durante cierto tiempo, moderado grado de infiltración leucocitaria alrededor.

\section{Cambios secundarios}

Placentitis: en general la encontramos en grado moderado, siendo más constante en la ruptura del seno mar- 
ginal, localizada en la decidua subcorial. Se considera como reacción secundaria a la presencia de hemorragia, la que según el grado de evolución va hacia la organización e infiltración leucocitaria. En cada una de estas entidades encontramos algunos casos de infección severa tanto a nivel de las membranas, del cordón umbilical y del tejido subcoriónico. Estos casos estaban asociados a impregnación de meconio y los consideramos secundarios a sufrimiento fetal e infección sobreagregada.

Infartos isquémicos: se clasificaron los casos que macroscópicamente se valoraron con $10 \%$ o más de superficie lesionada, ya que en todas las placentas, de embarazo a término, siempre presentan cierto número de infartos especialmente marginales.

En el presente trabajo encontramos el mayor número de casos con infartos isquémicos correspondientes a la placenta previa $31,8 \%$. El abruptio Placentae solo presenta el 22,2\%, dato contradictorio con el hallado en las diferentes estadísticas estudiadas que dan mayor porcentaje para el abruptio y nosotros consideramos en la misma forma, ya que esta entidad generalmente se asocia a entidades clínicas como la toxemia que produce un alto porcentaje de infartos placentarios. No tenemos explicación para este hallazgo.

Placenta circunvalada: encontramos dos casos. Esta entidad se considera según Williams como una falla en la implantación placentaria y según Bartholomew a desprendimientos placentarios o a hemorragia del seno marginal en los dos primeros trimestres del embarazo.

Corioangioma: Se encontró un caso de esta entidad. No consideramos que tenga alguna influencia sobre las entidades estudiadas.

\section{Manejo}

El Abruptio Placentae es considerado un accidente grave cuya naturaleza requiere o implica la terminación del embarazo sin dar lugar a optar por una conducta expectante.

Debemos diferenciar la conducta a seguir en estos casos, de acuerdo con la vitalidad fetal y la hemorragia genital presente. Nosotros pensamos, al igual que varios autores, que en los casos de feto muerto el tratamiento conservativo y el parto vaginal son preferibles a la operación cesárea, tanto por los riesgos inmediatos como tardíos que conlleva esta intervención sobre la paciente. Las pacientes con abruptio están generalmente intoxicadas, susceptibles al shock, y en general, son consideradas como un riesgo quirúrgico malo. Las medidas que deben tomarse están encaminadas a prevenir el shock y combatirlo rápidamente por transfusiones y/o sedación, fuera del tratamiento adecuado para la toxemia, si está presente. El trabajo debe ser inducido o estimulado con la amniotomía y la aplicación de mezclas de dextrosa en A.D. con pequeñas cantidades de pitocin.

Un cuello impreparado, que tenga una cierta longitud y pequeña o ninguna dilatación, no es contraindicación para un tratamiento conservativo y no constituye indicación para la cesárea. Estos cuellos casi siempre permiten la amniotomía. Su dilatación y borramiento se hacen con una facilidad sorprendente, bajo el efecto del trabajo natural 0 inducido.

En los casos con feto vivo y dilatación incipiente o escasa, la conducta preconizada es la cesárea.

No podemos hacer diferenciación entre el abruptio parcial o total, por cuanto a pesar de algunas diferencias 
clínicas, especialmente de tipo especulativo, no puede hacerse un pronóstico exacto, que ad vitam del feto.

En los casos en que la hemorragia genital es muy abundante o por efectos de la hipotensión y trombosis de los senos venosos, esta no se manifiesta exteriormente, sino por el signo de aumento de la altura uterina comprometiendo así la vida de la paciente y la integridad del útero, está justificada igualmente la conducta quirúrgica.

En los casos en que la hemorragia es indolora y el diagnóstico tentativo es de ruptura del seno marginal o de placenta marginal, debe hacerse un tratamiento conservativo y expectante, excepto en los casos en donde la hemorragia pueda comprometer la vida fetal o materna. Se recomienda la amniotomía.

Si la hemorragia ocurre demasiado temprano en el último trimestre y no permite un diagnóstico positivo de placenta previa, la pregunta que se hace el obstetra es, ¿hasta qué punto estaría justificado hacer una cesárea inmediata, solamente sobre la base de una hemorragia indolora y sin causa aparente? No debería ser así, por cuanto muchos de estos casos son observados después como rupturas del seno marginal.

Durante mucho tiempo se ha utilizado el tratamiento expectante apreciándose que no hay aumento de la mortalidad materna ni fetal, siempre y cuando haya facilidades para un manejo obstétrico inteligente y un adecuado reemplazo de sangre.

Si esto se aplica a la placenta previa, es por supuesto más aplicable a la ruptura del seno marginal, llevando a la paciente más cerca del término, permitiendo una mayor duración cer- vical y la posibilidad de confirmar o descartar un diagnóstico de placenta previa.

En el parto, bien sea por cesárea o por vía vaginal, la placenta debe recibirse con el menor trauma posible, con la idea de conservar las relaciones naturales de las membranas y su sitio de ruptura con la placenta, así como evitar alteraciones y disociaciones de coágulos adherentes, trombos y otras evidencias de hemorragia útero-placentaria. Esto será de gran ayuda para establecer el diagnóstico.

\section{Conclusiones}

De este estudio practicado sobre las hemorragias del último trimestre del embarazo, podemos concluir que el abruptio placentae, la placenta previa y la ruptura del seno marginal, son entidades con características propias y definidas, y por lo tanto, deben tenerse siempre en cuenta en el diagnóstico diferencial de los accidentes hemorrágicos de esta etapa de la gestación.

El signo primordial es la hemorragia, presente en el $75,2 \%$ de los casos y generalmente en cantidad moderada o escasa.

La cantidad promedio de hemorragia fue de 400 cc. en el abruptio y la placenta previa, mientras que en la ruptura del seno marginal la cantidad fue de 200 cc. o menos. En general engendran un buen pronóstico fetal y materno, siendo mejor éste en los casos de ruptura del seno marginal. Depende este pronóstico en gran parte, especialmente en lo que se refie$r$ al abruptio y la placnta previa, de la oportunidad del tratamiento. Estas entidades pueden considerarse como una complicación de la multiparidad y son causa frecuente de partos prematuros, $(41,4 \%)$. 
Es importante especialmente para el diagnóstico diferencial entre el abruptio y la ruptura del seno marginal, su asociación con la toxemiā, presente con la primera en el $74,2 \%$, mientras que con la segunda se asoció en el $26,8 \%$.

Las complicaciones del tipo de la hipofibrinogenemia y el shock, fueron escasas y solo se presentaron en los casos de abruptio y placenta previa.

La conducta varía en las diferentes entidades, siendo agresiva en el abruptio con feto vivo y en la placenta previa, mientras que debe ser expectante en el abruptio con feto muerto, la placenta marginal y la ruptura del seno marginal. Los cambios histopatológicos más importantes son: la presencia de coágulos marginales o retroplacentarios, la ruptura del seno marginal, los infartos hemorrágicos y el desgarro del tejido placentario. En lugar secundario deben analizarse los signos de placentitis e infartos isquémicos. Debe convertirse en rutina el manejo y examen cuidadoso de la placenta en todos los casos de hemorragia del último trimestre, para poder esclarecer la causa verdadera.

\section{Resumen}

Se estudiaron 118 casos de hemorragia del último trimestre del embarazo en el Instituto Materno Infantil "Concepción Villaveces de Acosta" de Bogotá.

Se comprueban los hallazgos de otros autores. Se analizan las secuencias má simportantes y se observan algunas similitudes, diferencias y relacinoes clínicas e histológicas. Se exponen algunos conceptos sobre el pronóstico feto-materno y algunas normas sobre el diagnóstico y manejo del abruptio placentae, placenta previa y ruptura del seno marginal de la placenta.

\section{BIBLIOGRAFIA}

1 ARCY L. B. Development Anatomy. W. B. Saunders \& Co. 1962.

2 BUMM ERNST. The development of the circulation of the maternal blood in the human placenta. Am. J. Obst. Gyn. 87.829. 1963.

3 BOURNE G. The Human amnion and Chorion. Year Book medical publishers. Inc. 1962.

4 CORNER GEORGE W. Exploring the placental maze. Am. J. Obst. \& Gyn. 86.408. 1963.

5 CROTTOGINI J. J., ZUBIAURRE L., CALDEYRO-BARCIA R. Circulación útero placentaria en el embarazo humano. Trabajo presentado al $4^{\circ}$ Congreso Mundial de la Federación de Ginecología y Obstetricia. Mar del Plata. Sep. 1964.

6 DE REZENDE J., NAHOUM J. C., BARCELLOS J. M., BELFORT P. La Placenta y los nuevos caminos de la Obstetricia. Rev. Sandorama. Julio 1965.

7 FERGUSON J. H., MILLER H. K. Toxemia of pregnancy at Charity Hospital in New Orleans. Surg., Gyn. \& Obst. 101: 257.-268. 1955.

8 FERGUSON J. H. Late Pregnancy bleeding and rupture of the marginal sinus, New England Journal of Medicine. Vol. 254, 14. 645. April 5, 1956.

9 FERGUSON J. H. Rupture of the marginal sinus of the placenta. J.A.M.A. Vol. 166, 5 : 476, 1958.

10 FERGUSON J. H., HATTON R. L. Abruptio placentae and rupture of the marginal sinus of the placenta. Some Relationships. Am. J. of Obst. \& Gyn. Vol. 78, 5: 947, 1959.

11 FISH J. S., BARTHOLOMEW R. A., COLVIN E. D., GRIMES W. H. The role of marginal sinus rupture in antenatal hemorrhage. Am. J. Obst. \& Gyn. Vol. 61. 1: 20, Jan. 1951.

12 GARNEY, H. L., DAVID G. F. Bleeding in the midtrimestre of pregnancy. Am. J. Obst. \& Gyn. 95: 430, 1966.

13 HARRIS B. A. Jr. Marginal placental bleeding. Am. J. Obst. \& Gyn. Vol. 64, 1: 61, 1952. 
Vol. $x X$

N" 6

14 NOVAK E., NOVAK E. Gynecologic and Obstetric Pathology. 4th. Edition. W. B. Saunders \& Co. 1958.

15 NOVAK - WOODRUFF. Gynecologic and Obstetric Pathology. 5th. Edition. W. B. Saunders \& Co. 1962.
16 SANCHEZ F. Causas del parto prematuro. Rv. Hospital San Juan de Dios. Bogotá. 7: 30,1963

17 VILLEE, C. A. The placental and fetal membranes. Chap. 3. The Williams and Wilkins Co., 1960. 